\title{
Personalidade e risco: estudo em finanças comportamentais
}

A tolerância ao risco é um fator importante que influencia uma ampla gama de decisões financeiras pessoais e é definida como a quantidade máxima de incerteza que alguém está disposto a aceitar ao se tomar uma decisão financeira ou a disposição de se envolver em comportamentos cujos resultados são incertos com possibilidade de se ter um resultado negativo. Este trabalho teve como objetivo analisar a relação entre tolerância ao risco e personalidade entre universitários. Para isto, foi desenvolvido e aplicado um questionário à 96 alunos e ex-alunos da PUC-Rio. O questionário baseou-se no teste Big Five de personalidade (GOLDBERG, 1990), no teste de gênero psicológico denominado Bem Sex Role Inventory (BEM, 1997) e na escala de tolerância ao risco de Grable e Joo (2004). Também foram coletados dados sócio-demográficos, tendo em vista as variáveis de: sexo, faixa etária, estado civil, escolaridade, composição familiar e renda familiar. Por meio de técnicas econométricas, foram desenvolvidos modelos nos quais o score de tolerância ao risco atua como variável dependente e as variáveis independentes são representadas pelo gênero psicológico, pela personalidade, na interação entre ambas e por dados sócio-demográficos. Os resultados apontaram que quanto maior os escores de gênero psicológico masculino e a estabilidade emocional maior a tolerância ao risco. Também foi observado que indivíduos do sexo masculino, com menor idade, que moram sozinhos, com nível de ensino superior e divorciados tem uma maior a tolerância ao risco.

Palavras-chave: Gênero Psicológico; Finanças Comportamentais; Personalidade; Tolerância ao Risco.

\section{Personality and risk: study in behavioral finance}

Risk tolerance is an important factor that influences a wide range of personal financial decisions and is defined as the maximum amount of uncertainty that someone is willing to accept to make a financial decision or the disposal of getting involved in behavior which results are uncertain with the possibility of having an negative result.This paper aims to analyze the relationship between risk tolerance and personality between university students. For this, we developed and applied a questionnaire to 96 students and former students from PUC-Rio. The questionnaire was based on Big Five test about personality (GOLDBERG, 1990), in the psychological gender test named Bem Sex Role Inventory (BEM, 1997) and in the risk tolerance scale from Grable and Joo (2004). Were also collected socio-demographic data, considering the variables of: gender, age, marital status, education, family composition and family income. Through econometric techniques, were developed models in which the score of risk tolerance was the dependent variable and the independent variables were represented by psychological gender, personality, the interaction between both and socio-demographics data. The results pointed that the higher the male psychological gender and emotional stability scores, the greater will be the risk tolerance.

Keywords: Psychological Gender; Behavioral Finance; Personality; Risk Tolerance.

\section{Topic: Finanças Empresariais}

Reviewed anonymously in the process of blind peer.

Heloisa lara Colchete Provenzano

Pontifícia Universidade Católica do Rio de Janeiro, Brasil http://lattes.cnpq.br/9723628083506607

heloicp@gmail.com

\section{Marcelo Cabus Klotzle}

Katholische Universitat Eichstatt, Alemanha

http://lattes.cnpq.br/5042677509706552

klotzle@iag.puc-rio.br

Paulo Vitor Jordão da Gama Silva

Pontifícia Universidade Católica do Rio de Janeiro, Brasil http://lattes.cnpq.br/5515359838087830

rjdagama@hotmail.com
Received: 12/06/2016

Approved: 23/11/2016
Jaysa Eliude Aguiar dos Santos

Universidade Federal da Paraíba, Brasil. http://lattes.cnpq.br/5401523574708658 jaysaeliude@yahoo.com.br

\section{Referencing this:}

PROVENZANO, H. I. C.; KLOTZLE, M. C.; SILVA, P. V. J. G.; PINTO, A. C. F.. Personalidade e risco: estudo em finanças comportamentais. Revista Brasileira de Administração Científica, v.7, n.3, p.39-51, 2016. DOI: http://doi.org/10.6008/SPC2179-684X.2016.002.0003 


\section{INTRODUÇÃO}

Ao longo dos últimos quarenta anos, houve um movimento crescente para entender melhor tolerância ao risco do ponto de vista de finanças domésticas e psicológica (DIXON et al., 1998). Há uma série de fatores que podem ser utilizados para compreender melhor tolerância ao risco, como os fatores sóciodemográficos, sócio-econômicos, comportamentais e psicológicos. Xiao (2008) destaca um modelo adaptado e modificado por Irwin (1993) que divide esses fatores em biopsicossociais (endógenos), ambientais (exógenos) e de precipitação (predisposição).

Assim, o objetivo deste trabalho é tentar entender como a personalidade, que será tomada pelos modelos como os de traços de personalidade do Big Five (GOLDBERG, 1990) e os traços de gênero psicológico no Bem Sex Role Inventory (BEM, 1997), se relacionam com o nível de tolerância ao risco. Para realizar esta análise, também serão coletados dados sócio-demográficos com o intuído de captar alguma tendência de comportamento tendo em vista as variáveis de sexo, faixa etária, estado civil, escolaridade, composição familiar e renda familiar.

Este é um tema relevante para a academia e para o mercado, pois existe uma carência de estudos similares no Brasil. É de suma importância na área de investimentos, uma vez que o mercado é formado principalmente por investidores individuais, que constantemente estão expostos a maior ou menor tolerância ao risco. Suas implicações vão desde um breve estudo sobre o tema até uma nova forma de se avaliar a tolerância ao risco de uma pessoa por meio de um questionário de personalidade.

Este artigo está dividido em: (2) referencial teórico envolvendo a revisão bibliográfica para dar suporte aos conceitos utilizados; (3) base de dados e a metodologia utilizada para a realização do estudo; (4) avaliação dos resultados e (5) considerações finais e sugestões para estudos futuros.

\section{REVISÃO TEÓRICA}

Para analisar a relação entre os traços de personalidade e a tolerância ao risco de um investidor foram utilizados modelos que capturam e medem estas informações. Um dos modelos utilizados foi o Big Five de personalidade (GOLDBERG, 1990), o Bem Sex Role Inventory de gênero (BEM, 1997) e escala de tolerância ao risco (GRABLE e JOO, 2004). Nesta seção, será feita uma revisão da literatura na qual serão abordados cada um destes modelos, bem como estudos anteriores sobre personalidade e risco.

\section{Modelo Big Five}

Segundo Goldberg (1993), o primeiro cientista a reconhecer que as diferenças individuais humanas poderiam ser codificadas como termos individuais em muitas línguas foi Sir Francis Galton, baseado em um pré-estudo de Thurstone, um legado de Cattell. Em 1884, Galton estimou o número de termos relacionados com a personalidade em Inglês. Estes termos foram revisados empiricamente em 1936 por Allport e Odbert e em 1967 por Norman, que identificou apenas cinco fatores replicáveis - por isso o nome Big Five. Outros 
estudos similares contendo também cinco fatores foram reportados como o FFM - Five Fator Model de personalidade.

A base teórica do Big Five advém da hipótese lexical - diferenças individuais que são mais saliente e socialmente relevantes e são codificadas como termos na língua natural. Já a base do FFM são os contextos teóricos, ou seja, os traços estão situados em um modelo abrangente de causas genéticas e ambientais e contextos. A nomeação dos fatores também diverge, bem como o questionário utilizado.

Os cinco traços de personalidade provaram ser robustos em diferentes países (ROBERTS e ROBINS, 2000), ser estável em toda vida adulta (MCCRAE e COSTA 2005) e prever uma série de resultados, tais como o desempenho no trabalho, o status ocupacional e o desempenho acadêmico, além de comportamentos e fenômenos (MALOUFF, THORSTEINSSON e SCHUTTE, 2006; SAULSMAN e PAGE, 2004; BARRICK, MOUNT e JUDGE, 2001). Os cinco traços de personalidade também estão relacionados ao julgamento e tomada de decisão em uma variedade de contextos, dentre eles as decisões financeiras e de risco (LAURIOLA e LEVIN, 2001; DURAND, NEWBY e SANGHANI, 2008; DURAND et al., 2013).

Em outras palavras, há evidências de que a personalidade, como esquematizada pelos cinco traços de personalidade, pode ser usada para explicar por que pessoas diferentes abordam determinadas tarefas e situações de maneiras diferentes. Por exemplo, pesquisadores demonstraram que os traços de personalidade amplos, como os incluídos no FFM de Costa e McCrae (1992) podem prever a propensão ao risco em uma variedade de situações e tarefas (GULLONE e MOORE, 2000; MARKEY et al., 2006).

Para este estudo, devido a facilidade de aplicação e maior utilização, optou-se por utilizar o modelo Big Five de Goldberg (1990). Os cinco traços de personalidade, do modelo em português são extroversão, amabilidade, conscienciosidade, estabilidade emocional e abertura para novas experiências.

A extroversão é caracterizada por emoções positivas e pela tendência em procurar estimulação e a companhia dos outros. Os extrovertidos são entusiastas, voltados para a ação, e geralmente aceitam oportunidades de excitação. Este traço possui um oposto, que são os introvertidos. Eles tendem a ser calmos, ponderados e menos envolvidos com o mundo social.

A amabilidade é a tendência em ser compassivo e cooperante em vez de suspeitoso e antagonista frente outros. Pessoas amigáveis têm também uma visão otimista da natureza humana. Elas acreditam que as pessoas são basicamente honestas, decentes e dignas de confiança.

A consciensciosidade é a tendência para mostrar autodisciplina, orientação para os deveres e para atingir os objetivos. Este traço mostra uma preferência pelo comportamento planejado em vez do espontâneo e influencia a maneira como são controlados e dirigidos os impulsos.

A estabilidade emocional caracteriza indivíduos que não se perturbam facilmente e são menos reativos. Este traço também tem um o oposto, que é o neuroticismo. Os neuróticos têm tendência a experimentar emoções negativas, como raiva, ansiedade ou depressão.

A abertura para novas experiências é o interesse pela arte, emoção, aventura, ideias fora do comum, imaginação, curiosidade e variedade de experiências. Este traço caracteriza pessoas intelectualmente curiosas, apreciadoras da arte, e sensíveis à beleza. 
O Big Five é aplicado aos entrevistados por meio de um questionário com cinquenta afirmativas (assim como pode ser notado na Tabela 8 - segunda parte do questionário, em anexo). 0 entrevistado atribui uma pontuação de 1 a 5 para cada afirmação de forma a indicar o quanto a mesma o descreve em comparação com outras pessoas de mesmo sexo e idade, sendo 1 - Absolutamente não me descreve, 2 - Me descreve mal, 3 - Me descreve parcialmente, 4-Me descreve bem e 5 - Me descreve perfeitamente. No final, é possível obter um escore para cada traço de personalidade do indivíduo.

\section{Modelo Bem Sex Role Inventory (BSRI)}

Bem (1981) assumiu o gênero como um constructo multidimensional e forneceu evidencias empíricas de que o gênero não necessariamente precisa estar atrelado ao sexo biológico. Ou seja, uma pessoa pode ter um alto ou baixo grau de masculinidade ou em feminilidade independente do sexo biológico. Desta forma, a referida autora desenvolveu uma escala de avaliação de gênero chamada Bem Sex-Role Inventory (BSRI) e criou o termo andrógeno para descrever os indivíduos que adotam tanto características masculinas quanto femininas.

Além disso, a autora propôs que uma pessoa mais equilibrada, capaz de incorporar ao mesmo tempo comportamentos masculinos e femininos, pode ser mais feliz e bem ajustada que alguém fortemente tipificado como puramente masculino ou feminino. A androginia oferece a vantagem de uma maior flexibilidade comportamental onde uma pessoa se move mais facilmente de uma situação para outra em sua vida. Enquanto isso, um autoconceito estritamente masculino pode inibir comportamentos que são estereotipados como femininos, e um autoconceito estritamente feminino pode inibir comportamentos que são estereotipados como masculinos.

Com isso, o BSRI passou a ser uma ferramenta importante em estudos envolvendo sexualidade e gênero. Hoje é utilizado como base para a avaliação do gênero em vários estudos de diferentes áreas. Em finanças comportamentais, Durand, Newby e Sanghani (2008) e Durand et al. (2013), por exemplo, utilizaram o gênero psicológico para identificar as diferenças entre os investidores e argumentaram que esta abordagem é melhor que aquela adotada por Barber e Odean (2001) que utilizaram somente o sexo biológico.

A escala original desenvolvida por Bem (1981) assume uma visão bidimensional do gênero com uma dimensão para medir a feminilidade e outra a masculinidade. Esta contém 20 traços masculinos e 20 femininos que foram percebidos como desejáveis por homens e mulheres, respectivamente, além de 20 traços neutros para evitar que os participantes fossem excessivamente influenciados por verem apenas traços femininos e masculinos. A escala também determina a androginia por meio da diferença entre as pontuações femininas e masculinas.

O entrevistado atribui a cada traço de personalidade uma pontuação de 1 a 7 , sendo: 1 = Nunca ou quase nunca é verdade; 2 = Normalmente não é verdade; 3 = Às vezes, mas raramente verdadeiro; 4 = Ocasionalmente verdadeiro; 5 = Muitas vezes é verdade; 6 = Geralmente é verdadeiro; e 7 = Sempre ou quase sempre verdadeiro. No final, soma-se a pontuação dos traços femininos e masculinos e divide-se cada uma 
por 10. Em seguida, é feita a subtração de ambos os resultados para determinar um escore. Os escores próximos a zero indicam a androginia, os escores que se afastam do zero no sentido positivo indicam feminilidade e os que se afastam no sentido negativo indicam a masculinidade. Também é possível utilizar apenas a soma das pontuações dos traços como escores variando de 10 a 70 cada um.

O uso frequente do BSRI na pesquisa de gênero e sua validade corroboraram para a sua aplicação neste estudo, contudo optou-se pela versão reduzida com 10 traços para cada classificação de gênero (BEM, 1997). Esta versão reduzida está na Tabela 9, no anexo e corresponde a fase 3 do questionário.

\section{Tolerância ao Risco}

Segundo Snelbecker, Roszkowski e Cutler (1990), a tolerância ao risco é um fator importante que influencia uma ampla gama de decisões financeiras pessoais. Muitos pesquisadores definem a tolerância ao risco como a quantidade máxima de incerteza que alguém está disposto a aceitar ao tomar uma decisão ou, segundo Irwin (1993), na disposição no qual um indivíduo se envolve em comportamentos cujos resultados são incertos com a possibilidade de um resultado negativo identificável.

Na prática, a tolerância ao risco tende a ser medida e avaliada por meio de um dos seis métodos: (a) julgamento pessoal ou profissional, (b) heurísticas, (c) objetivamente, (d) questões de um único item, (e) escalas de risco, ou (f) medidas mistas. (XIAO, 2008)

Para este estudo, o modelo utilizado para medir a tolerância ao risco foi a escala de risco de Grable e Joo (2004). Existem outras escalas mais tradicionais como o Jackson Personality Inventory (JACKSON, 1976) ou a escala do tipo likert de Weber e Betz (2002), contudo a primeira não está publicamente disponível e a segunda não é de fácil aplicação por ser longa.

A escala de risco de Grable e Joo (2004) é simples de se administrar, possui itens de validade razoável e fáceis de serem respondidos, fornece um escore em escala de tolerância ao risco e é relativamente atual. Esta foi usada pela primeira vez pelos referidos autores, quando foi testada usando uma amostra de 460 professores e funcionários de duas universidades norte-americanas do Meio-Oeste.

A escala contém cinco afirmativas e os entrevistados devem optar por uma das seguintes respostas para cada uma delas: (a) concordo totalmente, (b) tende a concordar, (c) tende a discordar, e (d) discordo fortemente. As respostas são codificadas em 1, 2, 3 ou 4, respectivamente, e somadas gerando um escore para cada participante. 0 escore médio para os respondentes foi de 12,86 , com um desvio padrão de 3,01 . A mediana da escala é 13,00 , o mínimo é 5,00 e o máximo é 20,00 . Os escores mais altos indicam maior tolerância ao risco.

A confiabilidade foi verificada com um Cronbach alpha de 0,8. Em relação a validade, as escalas foram associadas positivamente com a educação, o patrimônio, a renda familiar, o conhecimento financeiro, e a autoestima, conforme previsto na literatura de tomada de risco. A escala foi integrada no questionário aplicado à amostra e encontra-se na Tabela 10, no anexo deste trabalho, mais especificamente, na fase 4. 


\section{METODOLOGIA}

O questionário foi desenvolvido por meio da plataforma online Qualtrics e aplicado ao grupo de alunos e ex-alunos da PUC-Rio por meio da disponibilização do seu link por e-mail. No total foram 96 respondentes. A partir desses dados foram feitas a análise descritiva e a análise regressão da amostra por meio do software Eviews 7.0.

Conforme o objetivo deste estudo, a variável dependente é a tolerância ao risco e as variáveis independentes são as sócio-demográficas (faixa etária, sexo, estado civil, escolaridade, composição familiar e renda familiar); as de personalidade (abertura para novas experiências, conscienciosidade, estabilidade emocional, extroversão e amabilidade); e as de gênero (masculinidade, feminilidade e androginia).

A tolerância ao risco é uma variável numérica, assim como os traços de personalidade e o gênero. Ou seja, estas variáveis são representadas por uma pontuação ou escore. Já as variáveis sócio-demográficas são de caráter qualitativo ou categórico, ou seja, são representadas por palavras.

Para examinar o efeito de todas as variáveis independentes sobre a variável dependente a ser explicada foi utilizado o modelo de regressão linear múltipla. E para que fosse possível incluir as variáveis qualitativas ou categóricas na análise utilizou-se o modelo de regressão com variáveis dummies.

Na prática, foram utilizados três modelos de regressão linear múltipla. O Modelo 1 regrediu apenas as variáveis do gênero (masculinidade, feminilidade e androginia), em relação a tolerância ao risco. O Modelo 2 regrediu apenas as variáveis da personalidade (abertura para novas experiências, conscienciosidade, estabilidade emocional, extroversão e amabilidade), em relação a tolerância ao risco. Suas fórmulas são representas respectivamente pelas equações 1 e 2 :

$$
\begin{gathered}
Y_{\text {tolerância ao risco }}=\alpha+\beta_{1} X_{\text {masculino }}+\beta_{2} X_{\text {feminino }}+\beta_{3} X_{\text {androginia }}+\varepsilon \\
\quad \text { (Eq. 1) } \\
Y_{\text {tolerância ao risco }}=\alpha+\beta_{1} X_{\text {Abertura Novas Exp. }}+\beta_{2} X_{\text {Conscienciosidade }} \\
+\beta_{3} X_{\text {Estabilidade Emocional }}+\beta_{4} X_{\text {Extroversão }}+\beta_{5} X_{\text {Amabilidade }}+\varepsilon
\end{gathered}
$$

Já o modelo 3 regrediu as variáveis de gênero e de personalidade contra a tolerância ao risco (como pode ser visto na equação 3 ).

$$
\begin{aligned}
Y_{\text {tolerância ao risco }}= & \alpha+\beta_{1} X_{\text {Abertura Novas Exp. }}+\beta_{2} X_{\text {Conscienciosidade }}+\beta_{3} X_{\text {Estabilidade Emocional }} \\
& +\beta_{4} X_{\text {Extroversão }}+\beta_{5} X_{\text {Amabilidade }}+\beta_{6} X_{\text {masculino }}+\beta_{7} X_{\text {feminino }}+\beta_{8} X_{\text {androginia }}+\varepsilon
\end{aligned}
$$

(Eq. 3)

Também foi realizada regressão envolvendo dummies para avaliar as variáveis sócio-demográficas (sexo, a faixa etária, o estado civil, a escolaridade, a composição familiar e a renda familiar), que tiveram seu comportamento testado em relação à tolerância ao risco, assim como pode ser observado na equação 4 . 


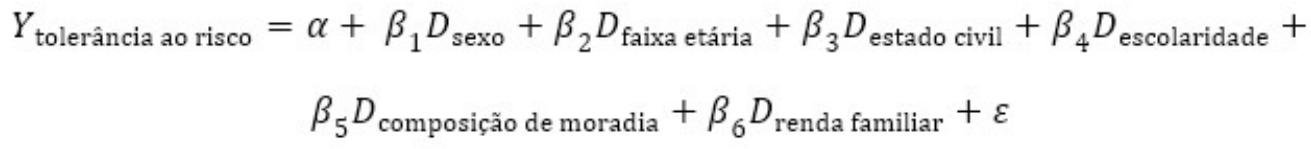

(Eq. 4)

\section{RESULTADOS}

O quadro 1 contém as informações descritivas da amostra. Os dados coletados foram classificados quanto a sua frequência em relação às variáveis sócio-demográficas.

Quadro 1: Estatística Descritiva - Variáveis Sócio-Demográficas.

\begin{tabular}{|l|l|l|}
\hline Faixa etária \\
\hline Menos de 25 anos & 5 & $5 \%$ \\
\hline Entre 25 e 45 anos & 68 & $71 \%$ \\
\hline Entre 46 e 65 anos & 22 & $23 \%$ \\
\hline Acima de 65 anos & 1 & $1 \%$ \\
\hline Total & 96 & $100 \%$ \\
\hline \multicolumn{2}{|l}{} \\
\hline Sexo & 52 & $54 \%$ \\
\hline Masculino & 44 & $46 \%$ \\
\hline Feminino & 96 & $100 \%$ \\
\hline Total
\end{tabular}

\begin{tabular}{|c|c|c|}
\hline \multicolumn{3}{|l|}{ Moradia } \\
\hline Sozinho (a) & 14 & $15 \%$ \\
\hline Com companheiro (a), outro familiar ou agregado (a) & 82 & $85 \%$ \\
\hline Total & 96 & $100 \%$ \\
\hline \multicolumn{3}{|l|}{ Estado Civil } \\
\hline Casado (a) ou em união consensual & 47 & $49 \%$ \\
\hline Solteiro (a) & 39 & $41 \%$ \\
\hline Divorciado (a) & 10 & $10 \%$ \\
\hline Total & 96 & $100 \%$ \\
\hline
\end{tabular}

\begin{tabular}{|l|l|l|}
\hline Escolaridade \\
\hline Ensino Superior & 15 & $16 \%$ \\
\hline Pós-graduação & 81 & $84 \%$ \\
\hline Total & 96 & $100 \%$ \\
\hline
\end{tabular}

\begin{tabular}{|l|l|l|}
\hline Renda & 1 & $1 \%$ \\
\hline Entre $\mathrm{R} \$ 678,01$ e $\mathrm{R} \$ 1.356,00$ & 2 & $2 \%$ \\
\hline Entre $\mathrm{R} \$ 1.356,01$ e $\mathrm{R} \$ 2.712,00$ & 93 & $97 \%$ \\
\hline Acima de $\mathrm{R} \$ 2.712,00$ & 96 & $100 \%$ \\
\hline Total & 96 \\
\hline
\end{tabular}

A amostra é composta, em maioria, por pessoas de 25 a 45 anos, com pós-graduação, que vivem com mais alguém e possuem renda acima de $\mathrm{R} \$ 2.712,00$. Em relação ao sexo, a amostra é composta por $54 \%$ de homens e $46 \%$ de mulheres, cujos $49 \%$ são casados, $41 \%$ solteiros e $10 \%$ divorciados. Esta analise descritiva condiz com a amostra obtida por conveniência por meio de alunos e alunos e ex-alunos da PUC-Rio. A Tabela 1 contém a estatística das variáveis de personalidade, gênero e risco.

Tabela 1: Estatística Descritiva - Variáveis Quantitativas.

\begin{tabular}{|c|c|c|c|c|c|c|}
\hline Modelos & Variáveis & Média & Mediana & Desvio Padrão & Mín. & Máx. \\
\hline & Ab.p/nv. exp. & 3,82 & 3,9 & 0,52 & 2,3 & 5 \\
\hline & Consc. & 3,82 & 3,8 & 0,59 & 2 & 5 \\
\hline \multirow[t]{4}{*}{ Traços de Personalidade } & Estab. Em. & 3,35 & 3,4 & 0,61 & 1,7 & 4,7 \\
\hline & Extroversão & 3,06 & 3,1 & 0,69 & 1,5 & 4,8 \\
\hline & Amabilidade & 3,79 & 3,9 & 0,59 & 1,9 & 4,7 \\
\hline & Masculinidade & 5,32 & 5,3 & 0,7 & 3,8 & 7 \\
\hline \multirow[t]{2}{*}{ Gênero Psicológico } & Feminilidade & 4,86 & 4,9 & 0,65 & 3,2 & 6,3 \\
\hline & Androginia & 4,61 & 4,6 & 0,43 & 3,7 & 5,8 \\
\hline Tolerância ao Risco & Tol. ao Risco & 13,15 & 13 & 3,04 & 7 & 20 \\
\hline
\end{tabular}

Os escores das variáveis de personalidade apresentaram média, moda e mediana similares, não havendo discrepância entre os traços de personalidade dentro da amostra. Já em relação ao gênero, a masculinidade teve um escore médio maior em comparação com os escores médios da feminilidade e da androginia. Na tolerância ao risco, o escore médio dos respondentes foi de 13,15, acima da mediana de 13,00. O escore mínimo da amostra foi de 7,00, acima do mínimo possível que é 5,00. 0 escore máximo foi 2,00, coincidindo com o máximo possível para essa variável. 
A Tabela 2 mostra o percentual de resposta de cada uma das afirmativas feitas para avaliar a tolerância ao risco. Tem-se que maioria tende a discordar que investir é de difícil compreensão, contudo grande parte sente-se mais confortável com o dinheiro em uma conta bancária que no mercado de ações. Uma linha tênue divide a opinião dos respondentes em relação a associar a perda ao risco. Além disso, maioria dos respondentes tende a discordar ou discorda plenamente que ganhar dinheiro em ações e títulos seja questão de sorte. Contudo, em termos de investimento, priorizam a segurança e não o retorno.

Tabela 2: Estatística Descritiva - Variáveis Quantitativas - Tolerância ao Risco.

\begin{tabular}{|c|c|c|c|c|c|}
\hline Questões Aplicadas & $\mathrm{CP}$ & $\mathrm{TC}$ & TD & $\mathrm{DP}$ & Total \\
\hline Investir é de difícil compreensão & $7,3 \%$ & $28,1 \%$ & $40,6 \%$ & $24,0 \%$ & $100 \%$ \\
\hline $\begin{array}{l}\text { Eu me sinto mais confortável colocando o meu dinheiro em uma conta bancária do que no mercado de } \\
\text { ações }\end{array}$ & $18,8 \%$ & $39,6 \%$ & $29,2 \%$ & $12,5 \%$ & $100 \%$ \\
\hline Quando eu penso na palavra risco, o termo perda vem logo em minha mente & $15,6 \%$ & $30,2 \%$ & $37,5 \%$ & $16,7 \%$ & $100 \%$ \\
\hline Ganhar dinheiro em ações e títulos é questão de sorte & $2,1 \%$ & $11,5 \%$ & $46,9 \%$ & $39,6 \%$ & $100 \%$ \\
\hline Em termos de investimento, considero segurança mais importante que retorno & $17,7 \%$ & $50,0 \%$ & $28,1 \%$ & $4,2 \%$ & $100 \%$ \\
\hline
\end{tabular}

Conforme a Tabela 3, a maior correlação positiva entre as variáveis é de 0,6 entre a feminilidade e a amabilidade. Já a maior correlação negativa de -0,4 ocorre entre as duas variáveis sócio-demográficas moradia e estado civil e entre tolerância ao risco e sexo. Segundo Callegari-Jacques (2003), o coeficiente de correlação pode ser avaliado qualitativamente da seguinte forma: se entre 0 e 0,3 , existe fraca correlação linear; se igual ou maior que 0,3 e menor que 0,6 , existe moderada correlação linear; se igual ou maior que 0,6 e menor que 0,9, existe forte correlação linear; e se igual ou maior que 0,9 e menor que 1 , existe correlação linear muito forte. Nesse sentido, pode-se dizer que a feminilidade e a amabilidade possuem forte correlação linear, enquanto a moradia e o estado civil e tolerância ao risco e sexo possuem correlação linear moderada.

Tabela 3: Análise de Correlação.

\begin{tabular}{|c|c|c|c|c|c|c|c|c|c|c|c|c|c|c|c|}
\hline & 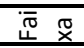 & $\cong 8$ & 䓌 $\bar{\sigma}$ & щّ $\overline{\mathrm{O}}$ & $\Sigma$ ò & $\underset{\propto}{\mathscr{q}} \bar{c}$ & $\frac{0}{4}$ & $8=$ & 岀 $\frac{0}{\pi}$ & ×인 & $<\varepsilon$ & 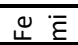 & $\sum n$ & $\frac{51}{4}$ & 을 \\
\hline Faixa etária & 1 & & & & & & & & & & & & & & \\
\hline Sexo & $-0,1$ & 1 & & & & & & & & & & & & & \\
\hline Estado Civil & $-0,2$ & 0,1 & 1 & & & & & & & & & & & & \\
\hline Escolaridade & 0,3 & 0,1 & $-0,1$ & 1 & & & & & & & & & & & \\
\hline Moradia & 0 & 0 & $-0,4$ & 0,1 & 1 & & & & & & & & & & \\
\hline Renda & 0 & $-0,1$ & 0 & 0 & 0 & 1 & & & & & & & & & \\
\hline Ab. p/ nv. Exp. & 0,1 & $-0,2$ & 0 & 0,1 & 0 & 0,1 & 1 & & & & & & & & \\
\hline Consc. & $-0,1$ & 0,1 & 0 & 0,1 & 0 & 0 & 0,3 & 1 & & & & & & & \\
\hline Estab. Em. & 0 & $-0,1$ & 0 & 0,1 & 0,1 & 0 & 0,3 & 0 & 1 & & & & & & \\
\hline Extroversão & 0,1 & 0,1 & 0,1 & 0,1 & 0 & 0 & 0,2 & 0 & 0,2 & 1 & & & & & \\
\hline Amabilidade & 0,2 & 0,2 & 0 & 0,1 & 0 & 0 & 0,3 & 0,2 & 0,2 & 0,2 & 1 & & & & \\
\hline Feminilidade & 0,1 & 0,2 & 0 & 0,1 & 0 & 0 & 0,2 & 0,1 & 0,3 & 0,3 & 0,6 & 1 & & & \\
\hline Masculinidade & 0 & $-0,2$ & 0,1 & 0,1 & $-0,1$ & 0,2 & 0,5 & 0,4 & 0,3 & 0,2 & 0,1 & 0,2 & 1 & & \\
\hline Androginia & 0 & 0 & 0,1 & $-0,2$ & $-0,1$ & $-0,1$ & 0 & 0,1 & $-0,2$ & 0,1 & 0 & 0,2 & 0,2 & 1 & \\
\hline Tol. ao Risco & $-0,1$ & $-0,4$ & 0,2 & 0 & $-0,3$ & 0,1 & 0,2 & 0 & 0,2 & 0 & $-0,1$ & 0 & 0,3 & 0,2 & 1 \\
\hline
\end{tabular}

A Tabela 4 contém os resultados da regressão realizada no Eviews 7.0. Conforme mencionado na metodologia, foram utilizados três modelos diferentes de regressão linear múltipla. O primeiro modelo considerou variáveis de gênero psicológico, o segundo considerou variáveis de personalidade e o terceiro, ambas.

Tabela 4: Resultado da Regressão. 


\begin{tabular}{|c|c|c|c|}
\hline $\begin{array}{l}\text { C } \\
\text { (p-valor) }\end{array}$ & $\begin{array}{l}4,74 \\
-0,22 \\
\end{array}$ & $\begin{array}{l}10,41^{* * *} \\
0\end{array}$ & $\begin{array}{l}2,92 \\
-0,51\end{array}$ \\
\hline $\begin{array}{l}\text { Masculinidade } \\
\text { (p-valor) }\end{array}$ & $\begin{array}{l}1,34 * * * \\
0 \\
\end{array}$ & & \begin{tabular}{|l|}
$1,24^{* *}$ \\
$-0,03$ \\
\end{tabular} \\
\hline $\begin{array}{l}\text { Feminilidade } \\
\text { (p-valor) }\end{array}$ & $\begin{array}{l}-0,52 \\
-0,28 \\
\end{array}$ & & $\begin{array}{l}-0,64 \\
-0,31 \\
\end{array}$ \\
\hline $\begin{array}{l}\text { Androginia } \\
\text { (p-valor) }\end{array}$ & $\begin{array}{l}0,82 \\
-0,26 \\
\end{array}$ & & $\begin{array}{l}1,46^{*} \\
-0,06\end{array}$ \\
\hline $\begin{array}{l}\text { Ab. p/ nv. Exp. } \\
\text { (p-valor) }\end{array}$ & & $\begin{array}{c}0,91 \\
-0,16 \\
\end{array}$ & $\begin{array}{c}0,31 \\
-0,65 \\
\end{array}$ \\
\hline $\begin{array}{l}\text { Consc. } \\
\text { (p-valor) }\end{array}$ & & $\begin{array}{l}-0,17 \\
-0,76 \\
\end{array}$ & $\begin{array}{l}-0,72 \\
-0,2 \\
\end{array}$ \\
\hline $\begin{array}{l}\text { Estab. Em. } \\
\text { (p-valor) }\end{array}$ & & $\begin{array}{l}1,15^{* *} \\
-0,03\end{array}$ & $\begin{array}{l}1,25^{* *} \\
-0,03\end{array}$ \\
\hline $\begin{array}{l}\text { Extroversão } \\
\text { (p-valor) }\end{array}$ & & $\begin{array}{l}-0,21 \\
-0,65 \\
\end{array}$ & $\begin{array}{l}-0,47 \\
-0,3 \\
\end{array}$ \\
\hline $\begin{array}{l}\text { Amabilidade } \\
\text { (p-valor) }\end{array}$ & & $\begin{array}{l}-0,88 \\
-0,11\end{array}$ & $\begin{array}{l}-0,31 \\
-0,64\end{array}$ \\
\hline $\mathrm{R} 2$ & 0,12 & 0,09 & 0,2 \\
\hline Prob. (Estatística F) & $0,01 * * *$ & 0,11 & $0,01 * * *$ \\
\hline
\end{tabular}

Fonte: Própria.

No modelo 1, que contém apenas as variáveis de gênero do BSRI, a regressão mostra uma relação positiva e significante à $1 \%$ da masculinidade com a tolerância ao risco. Ou seja, quanto maior o escore de masculinidade, maior a tolerância ao risco. Entretanto, não sugere relação significante com a feminilidade, nem com a androginia. $O \mathrm{R}^{2}$ obtido indica o quanto as variáveis independentes do modelo explicam a variável dependente. Nesse caso, as variáveis de gênero psicológico explicam $12 \%$ a tolerância ao risco. A estatística F é significante à $1 \%$ e indica que o modelo está bem ajustado.

O Modelo 2, que considera apenas as variáveis de personalidade, aponta para uma relação positiva e significante a $5 \%$ da estabilidade emocional com a tolerância ao risco. $O \mathrm{R}^{2}$ obtido indica que as variáveis de personalidade explicam $9 \%$ a tolerância ao risco. A estatística F é 11\%, o que indica que o modelo não é robusto, mas está bem ajustado.

No modelo 3, que inclui os traços de personalidade do Big Five além das variáveis de gênero, a regressão mostra uma relação positiva e significante à $10 \%$ da androginia e à $5 \%$ da masculinidade e da Estabilidade Emocional com a tolerância ao risco. Ou seja, todas estas três variáveis independentes influenciam positivamente a variável dependente. Se elas aumentarem, a tolerância ao risco também aumenta, e vice-versa. $O \mathrm{R}^{2}$ obtido indica o quanto as variáveis independentes do modelo explicam a variável dependente. Nesse caso, as variáveis de gênero psicológico explicam $20 \%$ a tolerância ao risco. A estatística F é significante a $1 \%$ confirmando que é um modelo bem ajustado e confirmando a significância do $R^{2}$ encontrado. Dados de caráter qualitativo ou categóricos também foram incluídos no modelo de regressão em forma de dummies com a finalidade de testar o comportamento dos mesmos em relação a tolerância ao risco (Quadro 2).

Quadro 2: Variáveis Dummies.

\begin{tabular}{|l|l|}
\hline Dummies - Escolaridade \\
\hline Ensino Superior & $13,47^{* * *}$ \\
(p-valor) & $(0,00)$ \\
\hline Pós-graduação & $13,09 * * *$ \\
(p-valor) & $(0,00)$ \\
\hline
\end{tabular}

\begin{tabular}{|l|l|}
\hline Dummies - Sexo & $14,21^{* * *}$ \\
\hline Masculino & $(0,00)$ \\
\hline (p-valor) & $11,89^{* * *}$ \\
\hline Feminino & $(0,00)$ \\
(p-valor) &
\end{tabular}




\begin{tabular}{|l|l|}
\hline Dummies - Moradia \\
\hline $\begin{array}{l}\text { Sozinho (a) } \\
\text { (p-valor) }\end{array}$ & $\begin{array}{l}15,14^{* * *} \\
(0,00)\end{array}$ \\
\hline $\begin{array}{l}\text { Com companheiro (a), outro } \\
\text { familiar ou agregado (a) } \\
\text { (p-valor) }\end{array}$ & $\begin{array}{l}12,80^{* * *} \\
(0,00)\end{array}$ \\
\hline
\end{tabular}

\section{Dummies - Faixa etária}

\begin{tabular}{|l|l|}
\hline $\begin{array}{l}\text { Menos de } 25 \text { anos } \\
\text { (p-valor) }\end{array}$ & $\begin{array}{l}15,60^{* * *} \\
(0,00)\end{array}$ \\
\hline $\begin{array}{l}\text { Entre 25 e 45 anos } \\
\text { (p-valor) }\end{array}$ & $13,03^{* * *}$ \\
& $(0,00)$ \\
\hline Entre 46 e 65 anos & $13,05^{* * *}$ \\
(p-valor) & $(0,00)$ \\
\hline Acima de 65 anos & $11,00^{* * *}$ \\
(p-valor) & $(0,00)$ \\
\hline
\end{tabular}

Em termos gerais, todas as variáveis dummies tem relação significativa com a tolerância ao risco. Verificou-se que quanto menor a idade, maior a tolerância ao risco. O resultado aponta também para maior tolerância ao risco entre homens que entre mulheres.

O mesmo ocorre com quem mora sozinho, que aparentemente é mais tolerante ao risco que aquele que mora acompanhado. Em relação a escolaridade, os que possuem nível superior e os pós-graduados têm praticamente a mesma relação positiva e significante com a tolerância ao risco. Já os divorciados e solteiros tem mais tolerância ao risco que os casados. A renda não foi incluída porque não apresentou variabilidade suficiente.

\section{DISCUSSÃO}

Os psicólogos Lauriola e Levin (2001) discutiram a diferença com que psicólogos de personalidade e pesquisadores de tomada de decisão estudavam a propensão ao risco. Eles fizeram uma pesquisa em uma amostra italiana de 78 respondentes, sendo 36 homens e 36 mulheres com faixa etária diversificada, para avaliar a relação entre os traços de personalidade, a idade, o gênero e a propensão ao risco. 0 resultado suportou a hipótese de relação entre os traços de personalidade e a propensão ao risco.

Durand, Newby e Sanghani (2008) fizeram um estudo para examinar se a personalidade determinava a decisão de investimento. Este estudo foi feito com uma amostra de 18 investidores individuais australianos. Para analisar a personalidade dos investidores foram utilizados os questionários Big Five de personalidade (NORMAN, 1963), o BRSI de gênero psicológico (BEM, 1977) e o Jackson Personality Inventory de preferência pela inovação ou pelo risco (JACKSON, 1976). Além disso, eles também coletaram algumas variáveis sóciodemográficas e a opinião sobre investimento dos investidores por meio de questões utilizadas no estudo de Bondt (1998).

Lee, Deck, Reyes e Rosen (2008) utilizaram dois experimentos pagos: o procedimento de Holt e Laury (2002) e uma variação do game show Deal or No Deal para medir atitudes de risco. Os participantes também completaram uma série de questionários de personalidade incluindo o FFM e uma escala de atitudes de risco, a fim de verificar se as diferenças individuais na personalidade e nas atitudes representam variação no desempenho em tarefas de tomada de decisões que envolvam risco. 
Os autores concluíram que os indivíduos assumiram o procedimento Holt e Laury (2002) como uma decisão de investimento, enquanto o Deal or No Deal como uma decisão de jogo. Os resultados sugeriram também que os indivíduos tendem a ser mais propensos ao risco no experimento Holt e Laury (2002) que no jogo Deal or No Deal. Em relação ao risco implícito, os indivíduos tendem a ser menos avessos ao risco do que eles reportaram previamente. Mas o parâmetro de risco dos dois experimentos não se comporta da mesma forma, alguns sujeitos alternam entre propensão e aversão ao risco e vice-versa.

Durand et al. (2013) apresentaram uma extensão de Durand, Newby e Sanghani (2008), reinterpretando sua questão central. No primeiro estudo foi demonstrado que a personalidade do investidor estava relacionada com suas escolhas de investimentos e os resultados. No segundo estudo, os autores confirmaram os resultados encontrados no primeiro. Além disso, sugeriram que investidores com traços de personalidade particulares definem o preço marginal de seguros com traços também particulares, pois a personalidade gera atração pelas características (ou traços) do seguro.

É possível perceber que todos os estudos analisados acima sugerem, de uma forma geral, a existência de uma relação estatisticamente significante entre a personalidade e o risco. Entretanto, existe divergência entre os traços de personalidade determinados como significantes. Por exemplo, Lauriola e Levin (2001) sugerem que a Estabilidade Emocional e a Abertura para Novas Experiências são os traços mais significantes, enquanto Durand, Newby e Sanghani (2008) e Durand et al. (2013) sugerem que a Estabilidade Emocional e a Extroversão o são. Apesar da Estabilidade Emocional ter sido apontada como significante em mais de um estudo, o sinal de sua significância também divergiu.

Em Lauriola e Levin (2001), maior escore em Estabilidade Emocional previu a aversão ao risco quando o indivíduo estava frente a uma possibilidade de ganho e previu a tolerância ao risco frente a uma possibilidade de perda. Já em Durand, Newby e Sanghani (2008), baixo escore em Estabilidade Emocional foi relacionado a portfolios com riscos mais altos. E, por fim, em Durand et al. (2013), baixo escore em Estabilidade Emocional foi relacionado a uma maior heurística disponível e esta, por sua vez, a uma menor propensão ao risco. Tais divergências podem existir pois os estudos diferem em relação as características da amostra, aos questionários aplicados, à metodologia utilizada e até mesmo quanto à análise dos resultados. Mas é importante ressaltar que apesar disso os estudos evidenciam a existência de uma relação estatisticamente significante entre traços de personalidade e tolerância ao risco.

Outra observação importante sobre os estudos analisados é a relação dos dados sócio-demográficos com os traços de personalidade e a tolerância ao risco. Em Lauriola e Levin (2001), a idade e o sexo biológico foram relacionados a cada um dos cinco traços de personalidade e a propensão ao risco. Foram encontradas relações significativas e isto levou os psicólogos a controlarem as regressões por estas variáveis sóciodemográficas.

\section{CONCLUSÃO}

Os resultados obtidos apontaram para a existência de uma relação significante entre a tolerância ao risco de um indivíduo e alguns de seus traços de personalidade utilizados na pesquisa. Isto equivale a dizer 
que a tolerância ao risco pode ser explicada em parte pela personalidade. Tais resultados confirmam, de uma forma geral, estudos anteriores, que atribuem à tolerância ao risco fatores biopsicossociais (endógenos), ambientais (exógenos) e de precipitação (predisposição).

O primeiro modelo estudou a relação entre a tolerância ao risco e os gêneros psicológicos. Este constatou a existência de uma relação estatisticamente significante positiva entre a masculinidade e a tolerância ao risco. Viu-se que quanto maior o escore de masculinidade, maior a tolerância ao risco.

O segundo modelo estudou a relação entre a tolerância ao risco e os traços de personalidade. Este constatou a existência de uma relação estatisticamente significante positiva entre a estabilidade emocional e a tolerância ao risco. Viu-se que quanto maior o escore de estabilidade emocional, maior a tolerância ao risco.

O terceiro modelo estudou a relação entre a tolerância ao risco com os gêneros psicológicos e os traços de personalidade. Os resultados reforçam o modelo 1, apontando uma relação estatisticamente significante positiva entre a masculinidade e a tolerância ao risco, bem como a androginia (porém com menos significância estatística que a masculinidade - isto ocorreu possivelmente devido a correlação entre os traços de gênero psicológico e de personalidade) e também confirma uma relação estatisticamente significante positiva entre a estabilidade emocional e a tolerância ao risco.

No que se referem as variáveis sócio-demográficas, foi observado que indivíduos do sexo masculino, com menor idade, que moram sozinhos, com nível de ensino superior e divorciados tem uma maior a tolerância ao risco.

Para trabalhos futuros, sugere-se a aplicação dos modelos de personalidade ligados à indicadores financeiros de desempenho de carteiras de ações de investidores, com o intuito de entender as relações com tais indicadores.

\section{REFERÊNCIAS}

BARBER, B. M.; ODEAN, T.. Boys Will Be Boys: Gender, Overconfidence, and Common Stock Investment. Quarterly Journal of Economics, v.116, n.1, p.261-292, 2001. DOI: http://doi.org/10.1162/003355301556400

BARRICK, M. R.; MOUNT, M. K.; JUDGE, T. A.. Personality and Performance at the Beginning of the New Millennium: What Do We Know and Where Do We Go Next?. International Journal of Selection and Assessment, v.9, n.1-2, p.9-30, 2001. DOI: http://doi.org/10.1111/1468-2389.00160

BEM, S. L.. Bem Sex-Role Inventory - BSRI. In: PFEIFFER, J. W.; JONES, J. E.. Reference Guide to Handbooks and Annuals. San Diego: University Associates, 1977.

BEM, S. L.. Gender Schema Theory: A Cognitive Account of Sex Typing. Psychological Review, v.88, n.4, p.354-364, 1981. DOI: http://doi.org/10.1037/0033-295x.88.4.354

BEM, S. L.. The Factor Structure of the Bem Sex-Role Inventory (BSRI): Confirmatory Analysis of Long and Short Forms. Educational and Psychological Measurement, v.57, n.1, p.118-124, 1997. DOI:

http://doi.org/10.1177/0013164497057001008

BONDT, W. F.. A Portrait of the Individual Investor. European Economic Review, v.42, n.3-5, p. 831-844, 1998. DOI: http://doi.org/10.1016/s0014-2921(98)00009-9

CALLEGARI-JACQUES, S. M.. Bioestatística: Princípios e Aplicações. 2 ed. Porto Alegre: Artmed, 2003.

COSTA, P. T.; MCCRAE, R. R.. NEO PI-R Professional Manual. Odessa: Psychological Assessment Resources, 1992.

DIXON, M. R.; HAYES, L. J.; REHFELDT, R. A.; EBBS, R. E. Possible Adjusting Procedure for Studying Outcomes of Risktaking. Psychological Reports, v.82, n.3, p.1047-1050, 1998. DOI: http://doi.org/10.2466/pr0.82.3.1047-1050

DURAND, R. B.; NEWBY, R.; SANGHANI, J. An Intimate Portrait of the Individual Investor. Journal of Behavioral Finance, v.9, n.4, p.193-208, 2008. DOI: http://doi.org/10.2139/ssrn.887441 
DURAND, R. B.; NEWBY, R.; PEGGS, L.; SIEKIERKA, M.. Personality. Journal of Behavioral Finance, v.14, n.2, p.116133, 2013. DOI: http://doi.org/10.2139/ssrn.1690902

GOLDBERG, L. R.. An Alternative "description of personality": The Big-Five factor structure. Journal of Personality and Social Psychology, v.59, n.6, p.1216-1229, 1990. DOI: http://doi.org/10.1037/0022-3514.59.6.1216

GOLDBERG, L. R.. The Structure of Phenotypic Personality Traits. American Psychologist, v.48, n.1, p.26-34, 1993. DOI: http://doi.org/10.1037/0003-066x.48.1.26

GRABLE, J. E.; JOO, S.. Environmental and biopsychosocial factors associated with financial risk tolerance. Financial Counseling and Planning, v.15, n.1, p.73-88, 2004.

GULLONE, E.; MOORE, S.. Adolescent Risk-taking and the Five-factor Model of Personality. Journal of Adolescence, v.23, n.4, p.393-407, 2000. DOI:

http://doi.org/10.1006/jado.2000.0327

HOLT, C. A.; LAURY, S. K.. Risk Aversion and Incentive Effects. American Economic Review, v.92, n.5, p.1644-1655, 2002. DOI: http://doi.org/10.2139/ssrn.893797

IRWIN, C.. Adolescence and risk taking: How are they related?. In: BELL, N. J.; BELL, R. W.. Adolescent risk taking. Newbury Park: Sage Publications, 1993.

JACKSON, D. N.. Jackson Personality Inventory JPI: Manual. Goshen: Research Psychologists Press, 1976.

LAURIOLA, M.; LEVIN, I. P.. Personality traits and risky decision-making in a controlled experimental task: an exploratory study. Personality and Individual Differences, v.31, n.2, p.215-226, 2001. DOI: http://doi.org/10.1016/s0191-8869(00)00130-6

LEE, J.; DECK, C.; REYES, J.; ROSEN, C.. Measuring Risk Attitudes Controlling for Personality Traits. Economics Research, Working Paper Series. Paper 46, 2008. DOI: http://doi.org/10.2139/ssrn.1148521
MALOUFF, J. M.; THORSTEINSSON, E. B.; SCHUTTE, N. S.. The five-factor model of personality and smoking: a metaanalysis. Journal of Drug Education, v.36, n.1, p.47-58, 2006. DOI: http://doi.org/10.2190/9ep8-17p8-ekg7-66ad

MARKEY, C. N.; MARKEY, P. M.; ERICKSEN, A. J.; TINSLEY, B. J.. Children's behavioral patterns, the Five-Factor model of personality, and risk behaviors. Personality and Individual Differences, v.41, n.8, p.1503-1513, 2006. DOI: http://doi.org/10.1016/i.paid.2006.06.007

MCCRAE, R. R.; COSTA, P. T.. Personality in Adulthood. 2 ed. New York: Guilford Press, 2005.

NORMAN, W. T.. Toward an Adequate Taxonomy of Personality Attributes: Replicated Factor Structure in Peer Nomination Personality Ratings. The Journal of Abnormal and Social Psychology, v.66, n.6, p.574-583, 1963. DOI: http://doi.org/10.1037/h0040291

ROBERTS, B. W.; ROBINS, R. W.. Broad Dispositions, Broad Aspirations: The Intersection of Personality Traits and Major Life Goals. Personality and Social Psychology Bulletin, v.26, n.10, p.1284-1296, 2000. DOI:

http://doi.org/10.1177/0146167200262009

SAULSMAN, L. M.; PAGE, A. C.. The five-factor model and personality disorder empirical literature: A meta-analytic review. Clinical Psychology Review, v.23, n.8, p.1055-1085, 2004. DOI: http://doi.org/10.1016/i.cpr.2002.09.001

SNELBECKER, G. E.; ROSZKOWSKI, M. J.; CUTLER, N. E.. Investors' Risk Tolerance and Return Aspirations, and Financial Advisors' Interpretations: A Conceptual Model and Exploratory Data. Journal of Behavioral Economics, v.19, n.4, p.377-393, 1990. DOI: http://doi.org/10.1016/00905720(90)90024-2

WEBER, E. U.; BETZ, N. E.. A Domain-specific Risk-attitude Scale: Measuring Risk Perceptions and Risk Behaviors. Journal of Behavioral Decision Making, v.15, n.4, p.263-290, 2002. DOI: http://doi.org/10.1002/bdm.414

$X I A O$, J. J.. Handbook of Consumer Finance Research. New York: Springer, Science+Business Media, LLC, 2008. 